OPEN

SUBJECT AREAS:

DEVELOPMENT

TAL EFFECTOR NUCLEASE

Received

14 August 2013

Accepted

16 October 2013

Published

5 November 2013

Correspondence and requests for materials should be addressed to

H.A. (asahara.syst@

tmd.ac.jp) or S.T. (takada-s@ncchd.go.

ip)

* These authors contributed equally to this work.

\section{Production of Sry knockout mouse using TALEN via oocyte injection}

Tomoko Kato ${ }^{*}$, Kohei Miyata ${ }^{1,2 *}$, Miku Sonobe 3 , Satoshi Yamashita' ${ }^{1}$, Moe Tamano' , Kento Miura ${ }^{4}$, Yoshiakira Kanai ${ }^{4}$, Shingo Miyamoto ${ }^{2}$, Tetsushi Sakuma ${ }^{5}$, Takashi Yamamoto ${ }^{5}$, Masafumi Inui', Takefumi Kikusui $^{3}$, Hiroshi Asahara ${ }^{1,6,7} \&$ Shuji Takada'

\begin{abstract}
'Department of Systems BioMedicine, National Research Institute for Child Health and Development, Tokyo 157-8535, Japan, ${ }^{2}$ Department of Obstetrics and Gynecology, Faculty of Medicine, Fukuoka University, Fukuoka 814-0180, Japan, ${ }^{3}$ Department of Animal Science and Biotechnology, Azabu University, Kanagawa 252-5201, Japan, ${ }^{4}$ Department of Veterinary Anatomy, The University of Tokyo, Tokyo $113-8657$, Japan, ${ }^{5}$ Department of Mathematical and Life Sciences, Graduate School of Science, Hiroshima University, Hiroshima 739-8526, Japan, ${ }^{6}$ Department of Systems BioMedicine, Graduate School of Medical and Dental Sciences, Tokyo Medical and Dental University, Tokyo $113-8510$, Japan, ${ }^{7}$ CREST, Japan Science and Technology Agency (JST), Saitama 332-001 1, Japan.
\end{abstract}

Recently developed transcription activator-like effector nuclease (TALEN) technology has enabled the creation of knockout mice, even for genes on the Y chromosome. In this study, we generated a knockout mouse for Sry, a sex-determining gene on the Y chromosome, using microinjection of TALEN RNA into pronuclear stage oocytes. As expected, the knockout mouse had female external and internal genitalia, a female level of blood testosterone and a female sexually dimorphic nucleus in the brain. The knockout mouse exhibited an estrous cycle and performed copulatory behavior as females, although it was infertile or had reduced fertility. A histological analysis showed that the ovary of the knockout mouse displayed a reduced number of oocytes and luteinized unruptured follicles, implying that a reduced number of ovulated oocytes is a possible reason for infertility and/or reduced fertility in the $\mathrm{KO}$ mouse.

$\mathrm{n}$ most mammalian species, sex is determined by the presence or absence of the $\mathrm{Y}$ chromosome. In mice, the Sry gene locates to the minimum sex-determining region of the murine $\mathrm{Y}$ chromosome ${ }^{1}$, is expressed in the male genital ridge at the time of sex determination ${ }^{2}$ and has been proven to be a sex-determining gene based on gain-of-function experiments, i.e., the overexpression of Sry in XX mice achieved with transgenic mouse technology reveals a male phenotype ${ }^{3}$. Also in humans, the $S R Y$ gene has been shown to play a pivotal role in sex determination: point mutations or deletions of the $S R Y$ gene are found in approximately $15 \%$ of XY females, and translocated $S R Y$ is found in the autosomes of most XX males ${ }^{4}$. Although there are a number of suggestive observations, it is important to confirm the function of Sry in vivo using loss-of-function analyses with targeted mutagenesis in order to examine whether Sry is the one and only sex-determining gene on the Y chromosome and to finally confirm the Sry gene as the sex-determining gene and provide an animal model of XY female syndrome. However, it is difficult to create knockout (KO) mice of Y-linked genes using conventional homologous recombination-based methods with embryonic stem (ES) cells, as the process requires an adequate length of specific sequences of homologous arms to construct a $\mathrm{KO}$ vector, and the $\mathrm{Y}$ chromosome is rich in repeats.

In 2013, Sung et al. ${ }^{5}$ first reported that KO mice can be produced using transcription activator-like effector nuclease (TALEN) technology without conventional homologous recombination-based methods. TALEN protein is an artificial sequence-specific endonuclease that contains Xanthomonas transcription activator-like effector (TALE) and a nuclease domain of FokI restriction endonuclease ${ }^{6}$. DNA binding domain of TALE consists of a tandem repeat of 33-35 amino acid motifs in which there are two critical adjacent amino acid pairs called a repeat variable diresidue (RVD) that determines the binding specificity for single nucleotide. There is a one-toone relationship between the RVD and its recognition nucleotide ${ }^{7,8}$. Using this code, a TALEN can be constructed with a DNA binding motif recognizing the desired nucleotide sequence ${ }^{6}$. When two TALENs are expressed in a cell and bind to the genome at an appropriate distance, called a spacer, the nuclease domain of FokI dimerizes and generates a double-strand break (DSB) within the spacer. The lesion is frequently repaired via nonhomologous end joining (NHEJ), an error-prone mechanism that results in the introduction of small insertion or deletion (indel) mutations. It has been reported that TALENs are useful for creating $\mathrm{KO}$ animals, such as fruitflies? silkworms $^{10}$, zebrafish ${ }^{11-14}$, Xenopus $^{15,16}$ and rats $^{17,18}$. Recently, it has been shown that TALEN technology can be 
ID1 AGdATGGAGGGCCATGTCAAGCGCCCCATGAATGCATTTATGGTGTGGTCCCGTGGTGAGAGGCACAAGTTGGCCCAGCAGAATCCCAGCATGCAAAA--TACAGAGATCAGCAAGCAGCTGGGATGCAGg wt ID2 AGCATGGAGGGCCATGTCAAGCGCCCCATGAATGCATTTATGGTGTGGTCCCGTGGTGAGAGGCACAAGTTGGCCCAGCAGAATCCCAGCATGCAAAA--TACAGAGATCAGCAAGCAGCTGGGATGCAGG Wt ID8 AGCATGGAGGGCCATGTCAAGCGCCCCATGAATGCATTTATGGTGTGGTCCCGTGGTGAGAGGCACAAGTTGGCCCAGCAGAATCCCAGCATGCAAAA--TACAGAGATCAGCAAGCAGCTGGGATGCAGG Wt ID3 AGdATGGAGGGCCATGTCAAGCGCCCCATGAATGCATTTATGGTGTGGTCCCGTGGTGAGAGGCACAAGTTGGCCCAGCAGAATCCCAGCATGCAAAATTTAACAGAGATCAGCAAGCAGCTGGGATGCAGG +2 bp

B

Sex: $M$ M F F F F F ID: SM $\begin{array}{lllllllllllllllll}1 & 2 & 3 & 4 & 5 & 6 & 7 & 8 & 9 & 10 & 11 \mathrm{NC}\end{array}$
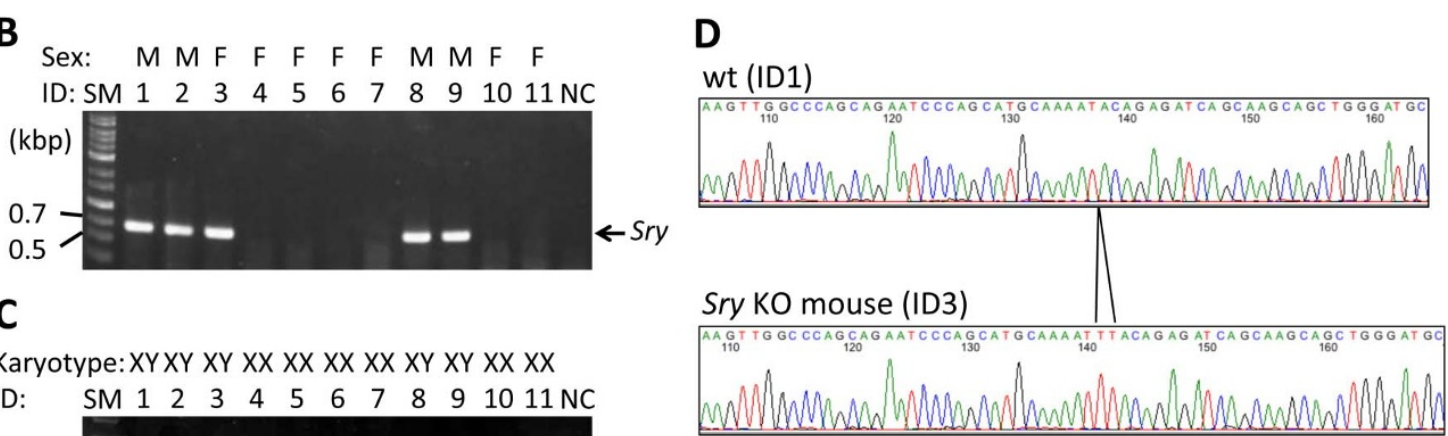

\section{C}

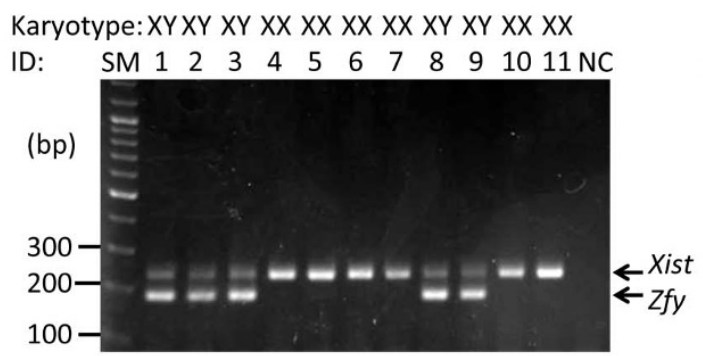

Figure 1 Construction of the TALEN and generation of the Sry KO mouse. (A) Summary of the TALEN design and genotyping of the KO mouse. The positions of the TALEN targets and spacer are indicated by green boxes and a green line, respectively, above the nucleotide sequences. The blue box indicates the start codon of the Srygene. ID of each mouse was shown at the left. Genotypes are indicated at the right. (B) Results of PCR amplification and agarose gel electrophoresis of Sry. M and F designate the phenotypic sex of male and female, respectively. DNA fragment lengths of the size markers are shown on the left. SM: size marker; NC: negative control. (C) Results of sexing PCR amplification and agarose gel electrophoresis. The karyotypes obtained from the experiment are shown at the top. DNA fragment lengths of the size markers are shown on the left. SM: size marker; NC: negative control. (D) Electropherograms around the TALEN spacer of the Sry KO (ID3) and wt male mice (ID1).

applied to generate autosomal gene $\mathrm{KO}$ mice via the microinjection of TALEN mRNA into fertilized oocytes ${ }^{5,19,20}$. TALEN can also be used to create $\mathrm{Y}$-linked gene $\mathrm{KO}$ mice, as it recognizes specific sequences as short as approximately 45-65 nucleotides long.

In 2012, Wu et al. ${ }^{21}$ reported the creation of a transgenic mouse in which Sry messenger RNA is knocked down in vivo using siRNA technology. In that report, the siRNA-treated developing gonads were feminized; however, it is difficult to knockdown target mRNA at $100 \%$ efficiency. Recently, the Sry gene was mutated using TALEN-mediated gene disruption in ES cells, and Sry KO mice were generated from the ES cells according to the tetraploid rescue method $^{22}$. The authors reported that the Sry KO mice possessed sex reversed internal and external genitalia. In the current study, we generated Sry KO mouse using the microinjection of TALEN RNA into fertilized oocytes and present a detailed analysis of the KO mouse in regard to the hormone levels, histology of the gonads and brain, as well as gross morphology.

\section{Results}

Construction of TALEN and the production of Sry KO mouse. In order to generate Sry KO mouse, we adopted the TALEN-mediated method instead of the conventional homologous recombinationbased ES cell modification strategy since Sry locates within $2.8 \mathrm{~kb}$ of a unique sequence at the center of a large inverted repeat structure $^{23}$. The TALEN-mediated method is suitable for gene disruption of such repeat embedded genes and can be used to more quickly obtain $\mathrm{KO}$ mice since it can be applied to microinjection into oocytes, thereby bypassing gene targeting and chimera mouse generation using ES cells. To disrupt the Sry gene using TALEN, we set the TALEN recognition sequence at the $5^{\prime}$ part of the open reading frame (ORF) (Fig. 1A), so that almost the entire protein of SRY was lost due to a frameshift mutation once the TALEN caused an indel mutation. The TALEN RNAs were first microinjected into fertilized oocytes, then cultured at $37^{\circ} \mathrm{C}$ until transferred into pseudopregnant female mice (78\% of the oocytes developed to the two-cell stage). PCR-sexing showed that 24 male pups were obtained out of 48 newborns, and PCR direct sequencing of Sry showed that no mutants were obtained (Table 1). It is possible that the optimal temperature for embryo culture, $37^{\circ} \mathrm{C}$, is not suitable for TALEN. Therefore, we changed the temperature for the embryo culture to $30^{\circ} \mathrm{C}$. This time, 129 oocytes were injected, $113(88 \%)$ of which entered two-cell stage embryos (Table 1); therefore, the lower

Table 1 | Disruption of the Sry gene using TALEN technology

\begin{tabular}{lcccrr} 
Temperature* & Injected & 2 -cell & Newborn & Males & Mutant \\
\hline $37^{\circ} \mathrm{C}$ & 130 & $101(78 \%)$ & $48(48 \%)$ & $24(50 \%)$ & $0(0 \%)$ \\
$30^{\circ} \mathrm{C}$ & 129 & $113(88 \%)$ & $23(20 \%)$ & $8(35 \%)$ & $1(13 \%)$ \\
\hline
\end{tabular}

The percentages shown in parenthesis were derived from the number in the column as the numerator and the number in the column to the left as the denominator times 100

*Temperature used for incubation after injection of the fertilized oocytes until transfer of the embryos into pseudopregnant females. 
temperature of the embryo culture did not appear to hamper development. However, the ratio of delivered pups was reduced compared to that obtained when the injected oocytes were cultured at $37^{\circ} \mathrm{C}$ (Table 1). Based on visual inspection of sexual dimorphism of the external genitalia and the existence of nipples in the resulting pups obtained from the injected oocytes cultured at $30^{\circ} \mathrm{C}$, we found that there were four males and seven females (Fig. 1B), whereas PCR genotyping in which the Zfy gene on the Y chromosome and the Xist gene on the $\mathrm{X}$ chromosome were amplified ${ }^{24}$ showed that five mice had a Y chromosome (Fig. 1C), indicating that there was a sex reversal mouse (XY female, ID3). To investigate the effects of the TALEN, the flanking sequences of the TALEN target site on the Sry gene were amplified and sequenced (Fig. 1A, B,D). Four XY males had no mutations, while ID3 had two nucleotide insertions in the ORF of the Sry gene, causing a frameshift mutation (Fig. 1A, D). Our loss-of-function experiment with the Sry gene confirmed that Sry is the sex-determining gene on the $\mathrm{Y}$ chromosome, consistent with the findings of previous reports ${ }^{3}$ utilizing gain-of-function experiments and the loss-of-function experiment of the Sry gene by Wang et al. ${ }^{22}$ utilizing ES cells and the tetraploid rescue method.

External genitalia of the Sry KO mouse. The phenotype of the Sry KO mouse was examined in detail. Wild type females obtained at the same time as the $\mathrm{KO}$ mouse via microinjection were used as controls. The external genitalia of the Sry KO mouse at one and two months after birth was indistinguishable from that of the wt females (Fig. 2A). To compare the external genitalia of the Sry KO mouse and wt females in detail, we set out to observe the timing of vaginal opening and measure the anal-genital distance (AGD), an index of femaleness. If the sexual differentiation of the Sry KO mouse is not complete, it is possible that the vagina of the KO mouse would not be open, the timing of vaginal opening would be delayed and/or the AGD would not differ between males and females. We checked the timing of vaginal opening in three-week-old mice. There was little difference between the $\mathrm{KO}$ and wt female mice. The AGD was measured and normalized according to body weight at four and eight weeks of age, at which time, the AGD/body weight of the KO mouse was in the range of that of the wt females (Fig. 2B, C). These results suggest that the Sry KO mouse had a completely female type of external genitalia.

Internal genitalia of the Sry KO mouse. We next investigated the internal genitalia of the Sry KO mouse. The ovaries, oviducts and uterus in the Sry KO mouse were similar in size and morphology to those of the wt females (Fig. 3A-D). To compare the structure of the ovaries of the Sry KO mouse and wt females in detail, ovary sections were stained with hematoxylin and eosin (Fig. 3E-H). A few developing follicles, such as secondary and Graafian follicles, were observed (Fig. 3E, F) in both the wt and Sry KO ovaries; however, the number of follicles in the Sry KO ovaries was approximately half of that of the wt ovaries (Fig. 3J), and luteinizing had proceeded in the KO ovaries. In addition, it is noteworthy that luteinized unruptured follicles (LUFs), including oocytes retained in the corpus luteum, were observed in the Sry KO ovaries (Fig. 3I) but not in the wt ovaries. These results suggest that the Sry KO mouse had internal female genitalia; however, the ovaries were not completely the same as those of the wt females.

Hormone levels and the estrous cycle of the Sry KO mouse. In order to examine whether the Sry KO mouse had female type endocrinological features, the estrous cycle was investigated by smearing the vagina every morning at eight weeks of age. The Sry $\mathrm{KO}$ mouse exhibited a proestrus period for two days (Fig. 4A, B), followed by estrus for three days (Fig. 4C-E), metaestrus for one day (Fig. 4F) and diestrus for one day (Fig. 4G). Subsequently, the estrus cycle turned back to the proestrus period (Fig. $4 \mathrm{H}$ ). The estrus period of the Sry KO mouse was a little longer than that of the wt females. Nonetheless, the estrus cycle of the Sry KO mouse was cycling. To confirm that the levels of sex hormones in the Sry KO mouse were in the range of females, the testosterone levels were measured. The blood plasma of four wt male mice obtained at the same time as the Sry KO mouse via microinjection, six wt females and the Sry
A

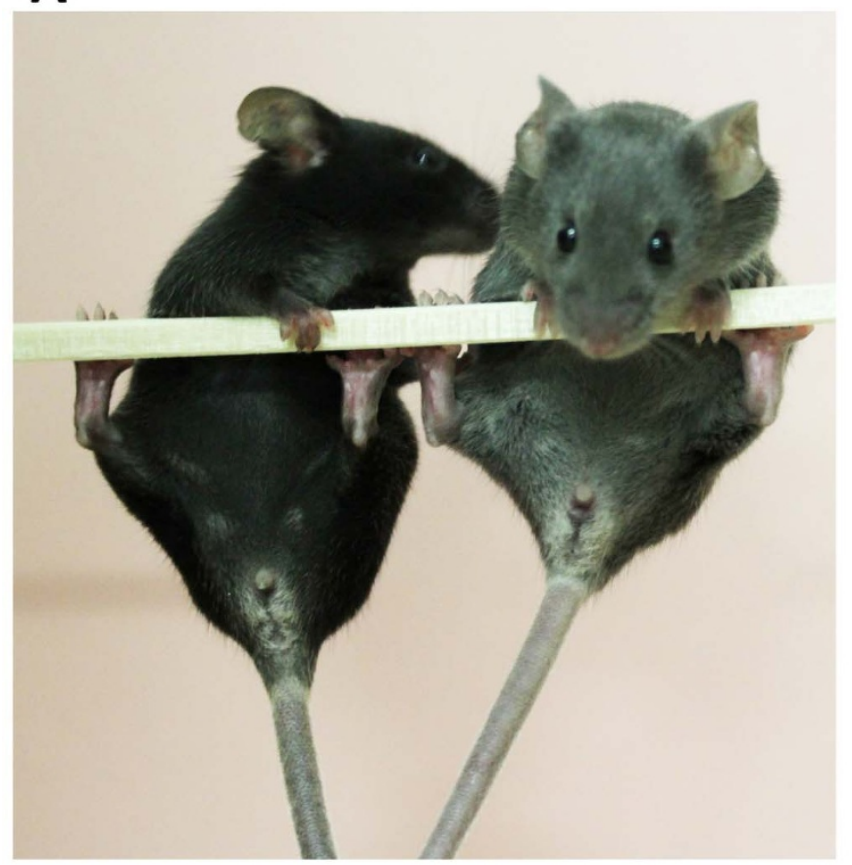

B
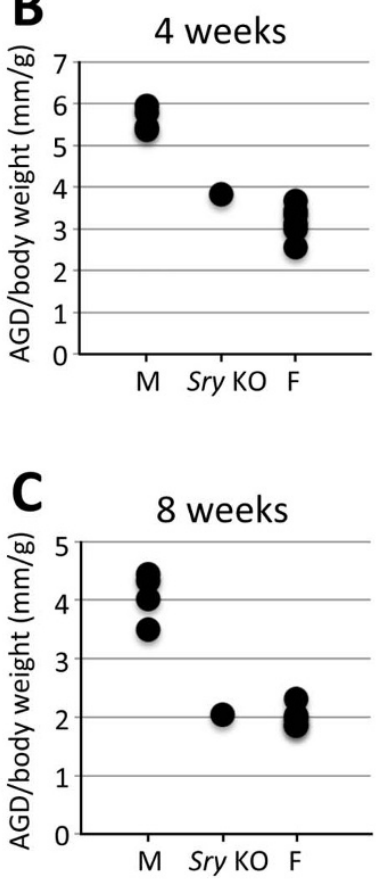

Figure $2 \mid$ External genitalia of the Sry KO mouse. (A) External genitalia of the Sry KO mouse. The photograph was taken in Animal Resources in National Research Institute for Child Health and Development by T.Kato and S.T. Left: Sry KO mouse; right: wt female mouse. (B) AGD normalized by body weight at four (top) and eight (bottom) weeks of age. Each dot shows the AGD/body weight of the individual mouse. M: male; F: female. 

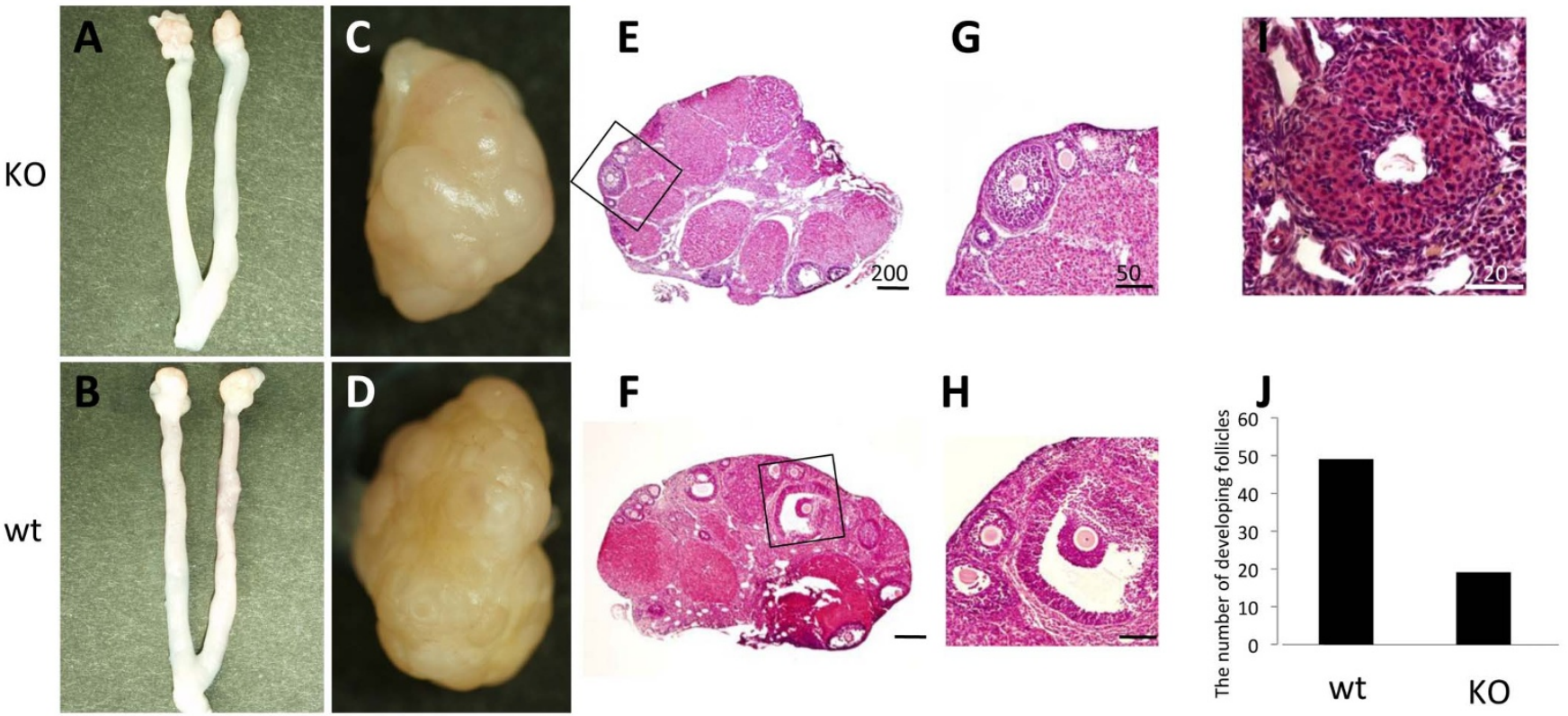

Figure 3 Internal genitalia of the Sry KO mouse. (A), (B) Ovaries and uterus of the Sry KO mouse (A) and wt female (B). (C), (D) Ovaries of the Sry KO mouse (C) and wt female (D). (E-H) Ovarian cross-section of the Sry KO mouse (E), (G) and wt female (F, H). G and H are higher magnification images of areas within the rectangles in E and F, respectively. Scale bars in E and F $=200 \mu \mathrm{m}$. Scale bars in G and H $=50 \mu \mathrm{m}$. (I) LUFs in the Sry KO ovary. Scale bar $=20 \mu \mathrm{m}$. (J) Total numbers of counted developing follicles in the Sry KO mouse and wt female.

KO mouse were used. The testosterone levels were high in the wt males, whereas the testosterone levels in the females, including the Sry KO mouse, were below the confident limit (the levels in the four wt females were below the measurement limit) (Fig. 4I). This result shows that the testosterone level of the Sry KO mouse was in the range of those of the wt females.
Fertility of the Sry KO mouse. To examine the fertility of the Sry KO mouse, Sry KO mouse (nine weeks old) were mated with wt C57BL/6 male mouse, of which the fertility was confirmed before and after the experiment by mating with wt females, for four weeks. Although we confirmed the presence of the vaginal plug four times, pregnancy was not observed in the KO mouse, as we did not find any newborn pups

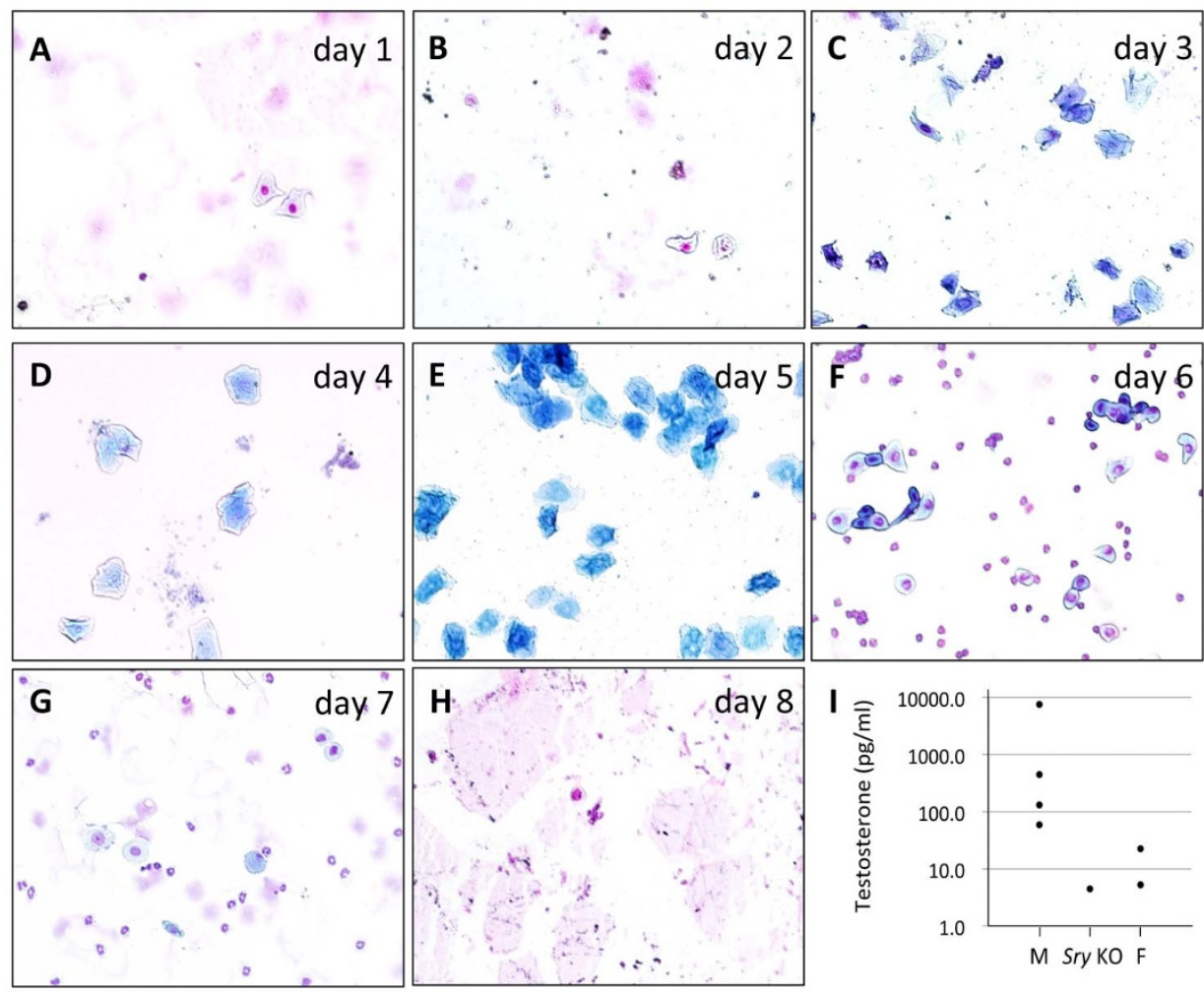

Figure $4 \mid$ Estrous cycle of the Sry KO mouse. Smear image of the Sry KO mouse. (A), (B), (H) Proestrus, (C-E) Estrus, (F) Metaestrus, (G) Diestrus. The day of the smear test is indicated on the top right in the image. (I) Testosterone levels of the Sry KO mouse. M: male; F: female. 
or embryos at dissection at 14 weeks of age. This result implies that the Sry KO mouse was sterile or had reduced fertility, even though it had oocytes in their ovaries and performed copulatory behavior as females.

Brain of the Sry KO mouse. The Sry KO mouse demonstrated reproductive behavior when mated with the wt males, which prompted us to confirm whether the KO mouse had female type sexually dimorphic nuclei. The presence of a sexually dimorphic nucleus was determined in the medial preoptic area (MPOA) using staining with calbindin, with a higher number of immunoreactive cells observed in males ${ }^{25}$. We found that the number of calbindin expressing neurons in the anteroventral periventricular nucleus (AVPV) in the MPOA was higher in the wt male compared to that observed in the wt female (Fig. 5). In the Sry KO mouse, the number of calbindin expressing cells was equivalent to that observed in the wt female and lower than that observed in the wt male, especially in the medial dorsal portion. However, a small number of calbindin positive cells was detected in the ventromedial preoptic area of the AVPV, a finding that was relatively comparable to that observed in the wt male (Fig. 5).

Effect of Sry mutation on gene expression. In the end, we wanted to confirm whether functional SRY protein production was truly disrupted in our mutant mouse. However, since we obtained only one mutant mouse which did not produce an offspring, detection of the SRY protein in embryonic gonad, where it acts as sex determination factor, was technically impossible. Therefore we expressed wt and mutant Sry gene in cultured cell to confirm whether two nucleotide insertion that we observed in Sry KO mouse disrupts SRY protein production. Overexpression vector encoding ORF of Sry gene with or without the two nucleotide insertion were transfected to HEK-293T and protein products were detected by western blotting (Fig. 6B-D). Since Sry is an intronless gene, Sry ORF was obtained from PCR amplification using genomic DNA as a template. To facilitate the detection and to clarify which part of the gene is produced as protein, FLAG and S-tag were fused to $\mathrm{N}$ - and C- termini of Sry, in the reading frame of wt Sry ORF (i.e. Cterminal S-tag of mutant Sry construct is out of frame to the first methionine) (Fig. 6A). As shown in Fig. 6B and 6C, SRY protein containing FLAG and S-tag were detected from wt Sry expressed cells, whereas no signal was detected from mutant Sry expressed cells. This result suggested that Sry mutation we obtained actually disrupts SRY protein expression. We presume there could be two reasons why we did not detect any protein product from mutant Sry construct: first, mutated Sry transcript has premature stop codon and thus was degraded by nonsense-mediated mRNA decay. Alternatively, truncated SRY protein was indeed synthesized, but the expected protein is short and could be structurally unstable, and thus degraded by unfolded protein response, or too small to detect western blotting.

\section{Discussion}

We generated Sry KO mouse using TALEN RNA injection into fertilized oocytes. KO mice have been generated using ES cells via homologous recombination, which requires targeting vectors containing the homologous arm of several kilobases. This hampers the creation of $\mathrm{KO}$ mice of genes that are in or around repeat-rich regions, such as Y-linked genes. On the other hand, TALEN requires relatively short specific sequences for genome editing so that it can be used to create $\mathrm{KO}$ mice for genes within repeat-rich regions. Another advantage in using TALEN to make $\mathrm{KO}$ mice is that it enables $\mathrm{KO}$ mice to be obtained quickly when injected into fertilize oocytes because the time required to culture ES cells and produce chimera can be omitted.

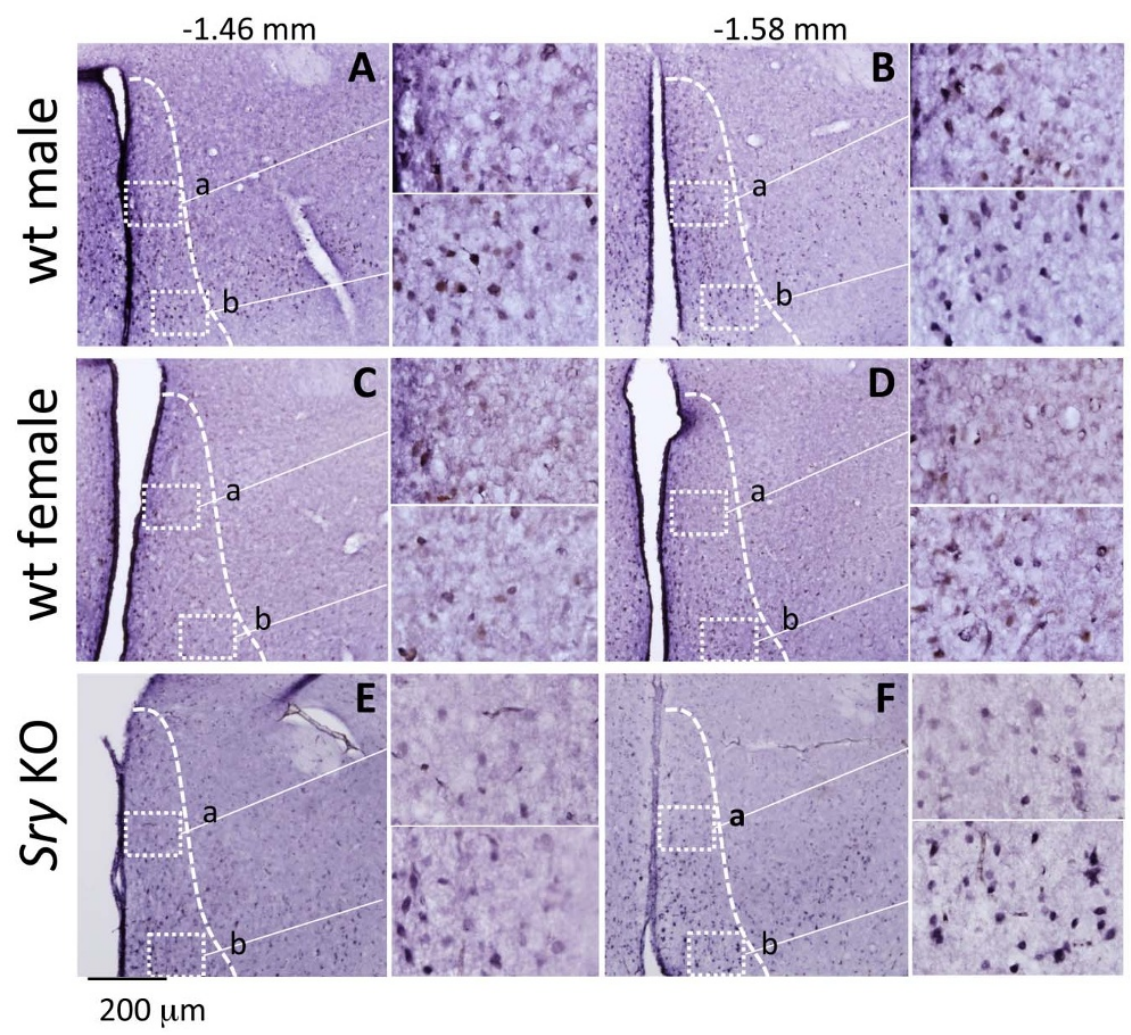

Figure $5 \mid$ Brain of the Sry KO mouse. Calbindin immunoreactive neurons were observed in the AVPV (dotted line) in the wt male (A, B), but not wt female (C), (D). The Sry KO mouse (E), (F) exhibited a lower expression level of calbindin in the medial dorsal portion of the AVPV compared to that observed in the wt male (square a); however, some positive cells were detected in the ventromedial preoptic area of the AVPV (square b). Scale bar $=200 \mu \mathrm{m}$. 


\begin{tabular}{l|l|l|l|}
\multicolumn{1}{c}{ mutant: } & FLAG & & \\
\hline
\end{tabular}

B

C

D
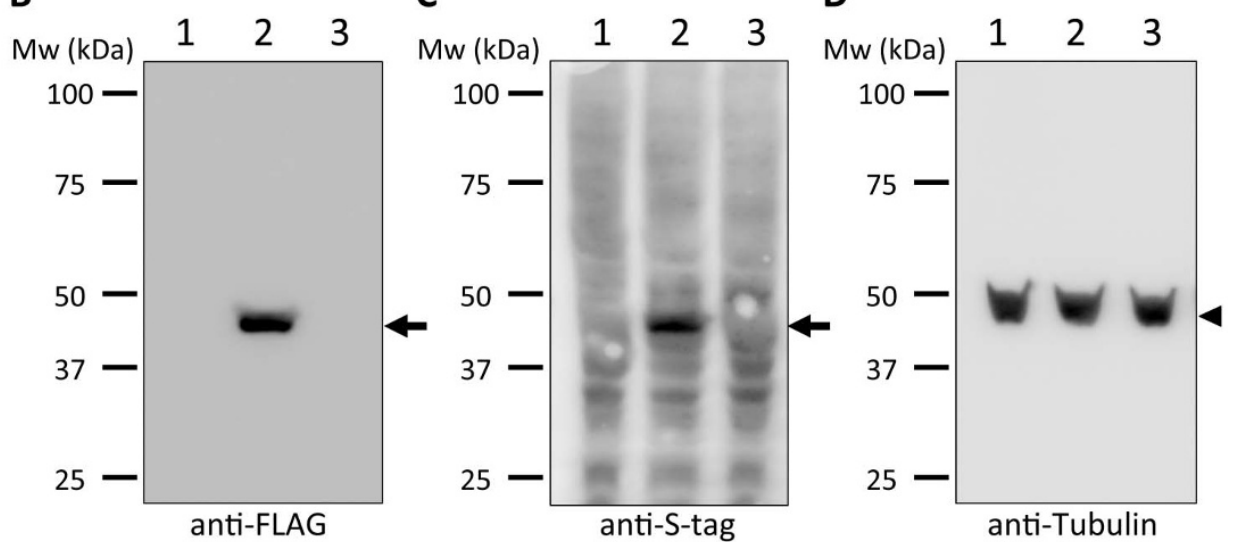

Figure $6 \mid$ Wild type and mutant Sry gene expression detected by western blotting. (A) Schematic representation of constructed gene structures in the expression vector. Position of the two nucleotide insertion (TT) is shown above the mutant gene structure. (B) Detection of wt and mutant SRY protein containing FLAG and S-tag at N- and C-termini, respectively, by western blotting using anti-FLAG antibody (arrow). Lane 1: empty vector transfected cell lysate; lane 2: wt Sry with FLAG and S-tag; lane3: mutant Sry with FLAG and S-tag. Size marker is shown at left of image. (C) Detection of wt and mutant SRY protein containing FLAG and S-tag at N- and C-termini, respectively, by western blotting using anti-S-tag antibody (arrow). Lanes and markers are same as those of (B). (D) Detection of tubulin by western blotting (arrowhead) as a control for amount of protein loading. Lanes and markers are same as those of $(B)$.

We could not obtain Sry KO mouse by incubation of TALEN RNA injected oocytes incubated at $37^{\circ} \mathrm{C}$. Since successful mutagenesis using TALEN was reported at the temperature lower than $37^{\circ} \mathrm{C}$ in heterothermic animals such as fruit flies ${ }^{9}$, silkworms ${ }^{10}$, zebrafish ${ }^{11-14}$ and Xenopus ${ }^{15,16}$, we thought lowering the incubation temperature could ameliorate the mutagenesis rate. Thus we incubate TALEN RNA injected oocytes at $30^{\circ} \mathrm{C}$ and could obtained the Sry KO mouse. We cannot conclude which temperature is better for making knockout mouse using TALEN from our data, but incubating injected oocyte at $30^{\circ} \mathrm{C}$ could be an option for whom cannot obtain knockout mouse when injected oocytes are incubated at $37^{\circ} \mathrm{C}$.

To evaluate sex reversal observed in Sry KO mouse is caused by Sry gene mutation, the best way is rescue of the phenotypes of the Sry KO mouse by crossing with Sry transgenic mouse. However this experiment could not be performed because the Sry KO mouse did not produce an offspring. We introduced a dinucleotide insertion at the 5' part of the ORF of the Sry gene, so that the protein product contains 32 intact amino acid residues (intact deduced SRY (RefSeq: NM_011564): 395 amino acid residues) with 30 new amino acids or mRNA is degraded by nonsense-mediated mRNA decay. In the former case the expected mutant SRY lacks functionally important motifs, such as a glutamine-rich domain, one of two nuclear localization signals (NLSs) and more than half of the HMG domain. Previous studies have reported that Sry transgenic constructs without the glutamine-rich domain fail to cause sex reversal in mice with the XX karyotype ${ }^{26}$, mutations in either NLS cause XY sex reversal in humans ${ }^{27-30}$ and most cases of human XY sex reversal involve mutations in HMG box (review in Harley and Goodfellow ${ }^{31}$ ). Considering these reports, it is reasonable that the Sry KO allele in our KO mouse does not produce functional SRY protein because $92 \%$ of the ORF was not translated and functionally important motifs were located on the missing residues.

In the present study, we analyzed the effects of Sry gene depletion in XY mouse. Recently Wang et a ${ }^{22}$ reported Sry KO mouse created through TALEN mediated gene disruption in ES cells. They showed the KO mouse had female type genitalia and reduced fertility. Our KO mouse, which had different mutant allele to previously reported mice, recapitulated the sex reversal phenotype which confirmed the fundamental role of Sry gene for male sex determination. Moreover, in this study we provided the histological and physiological phenotypes of Sry KO mouse. The external and internal genitalia were, as expected, female, the testosterone levels were within the female range, the estrous cycle was the same as that of the wt females, the copulatory behavior was the same as that of females and the dimorphic nucleus in the AVPV was of the female type. These results indicate that Sry KO mouse is sex reversed and healthy female, although the structure of the ovaries was different from that of normal females and the number of oocytes was reduced. We were unable to distinguish whether the Sry KO mouse had reduced fertility or was sterile, although we confirmed the presence of a vaginal plug four times, and no embryos or newborns were identified. Considering that Wang et al..$^{22}$ reported that Sry KO mice created using ES cell $\mathrm{KO}$ and tetraploid rescue have reduced fertility, it is possible that our $\mathrm{KO}$ mouse also had reduced fertility. If so, it is possible that the number of ovulated mature oocytes in the Sry KO mouse was reduced. Indeed, the ovaries of the Sry KO mouse contained fewer developing follicles and were more luteinized than those of the wt females. In addition, a few LUFs, including oocytes retained in the corpus luteum, were identified in the Sry KO ovaries only. In humans, LUFs are observed in 5-10\% of menstrual cycles of normal fertile females ${ }^{32}$, while a higher incidence of LUFs has been reported in infertile females ${ }^{33}$. In the latter cases, an estrous cycle is normal ${ }^{33-35}$. This situation is similar to that of the Sry KO mouse. This symptom of anovulation is considered to be a cause of human female infertility or reduced fertility ${ }^{35}$. However, we do not know whether the LUFs observed in the Sry KO mouse were caused by the existence of the $\mathrm{Y}$ chromosome or dysfunction of the ovaries. If the latter case is true, then it is possible that Sry KO mouse could be used as a model for infertility or reduced fertility containing LUFs. 
We found that the Sry KO mouse demonstrated female-like calbindin positive cells in the MPOA, especially in the medial dorsal portion of the AVPV. The distribution patterns of the calbindin expressing neurons in the wt males were different from the findings of a previous report of C57/BL6 males ${ }^{25}$. This is likely due to the difference in mouse strain $s^{36}$. In fact, clear sex differences in the number of calbindin expressing neurons were observed in the MPOA in this B6CBAF1/J strain in the medial dorsal portion of the AVPV, in which many Kisspeptin containing neurons exist. Kisspeptin neurons in the AVPV stimulate GnRH secretion ${ }^{37}$, which results in an increase in female copulatory behavior ${ }^{38}$. Interestingly, the Sry KO mouse exhibited female-like calbindin expressing neurons in the AVPV, suggesting that this female-like AVPV structure is related to the normal female-type copulatory behavior observed in the Sry KO mouse. However, a small number of calbindin positive cells was detected in the ventromedial preoptic area of the AVPV in the Sry KO mouse, which was relatively comparable to that observed in the wt male (Fig. 6). The neurons in the ventromedial preoptic area of the AVPV are related to the onset of anestrous in ewes ${ }^{39}$ and project to and stimulate hypothalamus dopaminergic neurons $\mathrm{s}^{40}$. A higher level of dopaminergic activity blocks pregnancy by decreasing the release of prolactin from the pituitary ${ }^{41}$. Therefore, the male-like structure of the ventromedial preoptic area of the AVPV may be related to the prolonged estrous cycle and reduced fertility observed in the Sry KO mouse. It is unclear why MPOA was not fully reversed from a male- to female-type structure in the Sry KO mouse. One possibility is a parent-of-origin imprinting gene expression in the hypothalamus. In the hypothalamus, sex-specific imprinted genes are found in females, which suggests a parental allelic influence over the hypothalamic function in XX females ${ }^{42}$. Therefore, Sry $\mathrm{KO}$ mouse lacked some female functions in the hypothalamus.

\section{Methods}

TALEN. The TALEN plasmids were designed using the online TAL Effector Nucleotide Targeter 2.0 software program (https://tale-nt.cac.cornell.edu/node/add/ talen-old) with the following parameters: Minimum Spacer Length at $12 \mathrm{bp}$, Maximum Spacer Length at 16 bp, Minimum Repeat Array Length at 16 and Maximum Repeat Array Length at 20. The query sequence used was the reverse complementary sequence of nucleotide number 1919368-1919568 on chromosome Y (Assembly by July 2007 (NCBI37/mm9)). Position 1 of the query sequence corresponds to the initiation codon of the Sry gene. One of the possible TALEN target sequences identified by the program was selected empirically. The TALENs were assembled in pcDNA-TAL-NC vector plasmids using a previously described protocol $^{43}$. A Golden Gate TALEN and TAL Effector Kit was obtained from Addgene ${ }^{44}$. The target sequences of the Sry TALENs were as follows: left 5' -TGG CCC AGC AGA ATC CCA GCA-3' and right 5' -TCC CAG CTG CTT GCT GAT- $3^{\prime}$

Microinjection. TALEN plasmids were digested by PvuII restriction endonuclease. One microgram of the digested plasmids was used as a template for the in vitro transcription reaction using the mMESSAGE mMACHINE T7 Kit (Life Technologies) according to the manufacturer's instructions. The synthesized RNAs were purified using the MegaClear kit (Life Technologies) according to the manufacturer's instructions. The RNA concentration was determined using a NanoDrop 1000 spectrophotometer and diluted with injection buffer (10 mM Tris$\mathrm{HCl} / 0.1 \mathrm{mM}$ EDTA (pH 7.4)) at $600 \mathrm{ng} / \mu \mathrm{l}$ in a total of two TALEN mRNAs $(1: 1$ ratio, i.e. $300 \mathrm{ng} / \mu \mathrm{l}$ each). The microinjection of the two TALEN mRNAs mix into cytoplasm of pronuclear stage oocytes was carried out under standard procedures using oocytes obtained from superovulated $(\mathrm{C} 57 \mathrm{BL} / 6 \times \mathrm{DBA} 2) \mathrm{F} 1$ mice mated with male mice of the same strain (Sankyo Labo Service Corporation). The injected oocytes were cultured in M16 medium at 30 or $37^{\circ} \mathrm{C}$. The following day, embryos developed into the two-cell stage were transferred into pseudopregnant ICR female mice. All animal care protocols and experiments were approved by the Animal Care and Use Committee at the National Research Institute for Child Health and Development.

PCR-based genotyping and sexing. Genomic DNA was extracted from tail tips using amputation. For genotyping of Sry, PCR was carried out using the following primers: SryF475 (5' - CTG TCC CAC TGC AGA AGG TT -3') and SryR412 (5' - GGG CTG GAC TAG GGA GGT CCT G - $3^{\prime}$ ). The PCR products were treated with ExoSAP-IT (Affymetrix) and used as templates for sequencing. The sequencing primer was SrySeq476 (5' - CAG CCC TAC AGC CAC ATG AT -3'). For PCR-based sexing, the $\mathrm{Y}$ chromosome-specific gene, Zfy, and the X chromosome-specific gene, Xist, were amplified using the primer set described by Obata et al. ${ }^{24}$.
Vaginal smear test. The estrous cycle was analyzed using a vaginal smear test. Sry KO mouse and wt females (eight weeks of age) were used for eight successive days. Every morning, PBS was applied to the vagina and pipetted well. The liquid was collected and uniformly smeared on a slide glass. The samples were air dried, fixed in methanol, stained with Giemsa solution and washed in tap water. The specimens were micrographed using an optical microscope.

Fertility check. Female mice nine weeks of age were mated with wt C57BL/6 male mice, of which fertility was confirmed by crossing with C57BL/6 females before and after crossing with the Sry KO mouse. The presence of the vaginal plug and parturition were checked every morning for four weeks.

Hormone assay. The mice were anesthetized, and blood was collected from the heart before euthanasia. The blood plasma was separated immediately after collection and stored at $-80^{\circ} \mathrm{C}$. The hormones levels were analyzed using a radioimmunoassay which was performed by Asuka Pharmaceutical Co., Ltd.

Histology. The mice were perfusion fixed through the heart using $4 \%$ paraformaldehyde. The brains and gonads were collected and embedded in paraffin. The embedded ovary paraffin block was sectioned at $6 \mu \mathrm{m}$. Deparaffinized sections were stained with hematoxylin and eosin. Six representative sections with more than $75 \mu \mathrm{m}$ of distance between each other were chosen, and the numbers of developing follicles, including secondary follicles and Graafian follicles, per ovarian cross section were counted. As to calbindin immunohistochemistry, sections containing the MPOA ( -0.5 to $-1.8 \mathrm{~mm}$ from bregma) were sectioned at $30 \mu \mathrm{m}$. One of two sequential sections was subjected to immunohistochemistry. The staining methods have been previously described ${ }^{25}$. Briefly, the sections were incubated with the first monoclonal antibody to calbindin (C9848-2ML; Sigma-Aldrich, $1: 12000)$ for 48 hours. After rinsing, the sections were incubated with biotinylated horse antimouse IgG antibodies (BA-2000, Vector Lab, 1:500), then reacted with the ABC kit (VECTASTAIN Elite ABC kit, Vector Labs).

Transfection and western blotting. ORF of Sry was PCR amplified using genomic DNA purified from wt male and Sry KO mouse using primers SryORF XhoI-FLAG F (5' - gcc tcg agA TGG ACT ACA AGG ACG ATG ATG ACA AGG GCa tgg agg gcc atg tca agc $\mathrm{g}-3^{\prime}$ ), where XhoI recognition site and FLAG tag indicated with underline and capital letters, respectively, and SryORF EcoRI StagR (5' - gcg aat tct caG CTG TCC ATG TGC TGT CTC TCG AAC TTG GCA GCG GCG GTC TCC TTt gag act gcc aac cac agg g- $3^{\prime}$ ), where EcoRI recognition site and FLAG tag indicated with underline and capital letters, respectively. PCR products were cloned in pIRES2EGFP (Clontech) using EcoRI/XhoI. Plasmids were transfected to HEK-293T cell using Fugene HD (Promega) according to the manufacturer's instruction. After 48 hours incubation at $37^{\circ} \mathrm{C}$, transfection efficiency was confirmed by checking EGFP emission and cells were lysed with cell lysis buffer (50 mM HEPES [pH 7.8], $200 \mathrm{mM} \mathrm{NaCl}, 5 \mathrm{mM}$ EDTA, 1\% NP40, 5\% glycerol, freshly complemented with $1 \mathrm{mM}$ DTT, protease inhibitor cocktail (Roche)). The cell lysate was analyzed by western blotting using 10\% SDS-PAGE, Immobilon PVDF memmbrane (Millipore), monoclonal anti-FLAG M2 antibody (SIGMA), anti-S-tag antibody (Abcam), antimouse IgG HRP conjugated (SIGMA), anti-rabbit IgG HRP conjugated (SIGMA) and ECL (GE).

1. Gubbay, J. et al. A gene mapping to the sex-determining region of the mouse $\mathrm{Y}$ chromosome is a member of a novel family of embryonically expressed genes. Nature 346, 245-250 (1990).

2. Koopman, P., Münsterberg, A., Capel, B., Vivian, N. \& Lovell-Badge, R. Expression of a candidate sex-determining gene during mouse testis differentiation. Nature 348, 450-452 (1990).

3. Koopman, P., Gubbay, J., Vivian, N., Goodfellow, P. \& Lovell-Badge, R. Male development of chromosomally female mice transgenic for Sry. Nature 351, 117-121 (1991).

4. Hawkins, J. R. et al. Mutational analysis of SRY: nonsense and missense mutations in XY sex reversal. Hum Genet 88, 471-474 (1992).

5. Sung, Y. H. et al. Knockout mice created by TALEN-mediated gene targeting. Nat Biotechnol 31, 23-24 (2013).

6. Christian, M. et al. Targeting DNA double-strand breaks with TAL effector nucleases. Genetics 186, 757-761 (2010).

7. Boch, J. et al. Breaking the code of DNA binding specificity of TAL-type III effectors. Science 326, 1509-1512 (2009).

8. Moscou, M. J. \& Bogdanove, A. J. A simple cipher governs DNA recognition by TAL effectors. Science 326, 1501 (2009).

9. Liu, J. et al. Efficient and specific modifications of the Drosophila genome by means of an easy TALEN strategy. J Genet Genomics 39, 209-215 (2012).

10. Ma, S. et al. Highly efficient and specific genome editing in silkworm using custom TALENs. PLoS One 7, e45035 (2012).

11. Huang, P. et al. Heritable gene targeting in zebrafish using customized TALENs. Nat Biotechnol 29, 699-700 (2011).

12. Sander, J. D. et al. Targeted gene disruption in somatic zebrafish cells using engineered TALENs. Nat Biotechnol 29, 697-698 (2011).

13. Bedell, V. M. et al. In vivo genome editing using a high-efficiency TALEN system. Nature 491, 114-118 (2012). 
14. Cade, L. et al. Highly efficient generation of heritable zebrafish gene mutations using homo- and heterodimeric TALENs. Nucleic Acids Res 40, 8001-8010 (2012)

15. Lei, Y. et al. Efficient targeted gene disruption in Xenopus embryos using engineered transcription activator-like effector nucleases (TALENs). Proc Nat Acad Sci U S A 109, 17484-17489 (2012).

16. Suzuki, K. I. et al. High efficiency TALENs enable F0 functional analysis by targeted gene disruption in Xenopus laevis embryos. Biology Open 2, 448-452 (2013).

17. Tesson, L. et al. Knockout rats generated by embryo microinjection of TALENs. Nat Biotechnol 29, 695-696 (2011).

18. Mashimo, T. et al. Efficient gene targeting by TAL effector nucleases coinjected with exonucleases in zygotes. Sci Rep 3, 1253 (2013).

19. Davies, B. et al. Site Specific Mutation of the Zic2 Locus by Microinjection of TALEN mRNA in Mouse CD1, C3H and C57BL/6J Oocytes. PLoS One 8, e60216 (2013)

20. Qiu, Z. et al. High-efficiency and heritable gene targeting in mouse by transcription activator-like effector nucleases. Nucleic Acids Res 41, e120 (2013).

21. Wu, N., Yu, A.-B., Zhu, H.-B. \& Lin, X.-K. Effective silencing of Sry gene with RNA interference in developing mouse embryos resulted in feminization of XY gonad. J Biomed Biotechnol 2012, 343891 (2012).

22. Wang, H. et al. TALEN-mediated editing of the mouse Y chromosome. Nat Biotechnol 31, 530-532 (2013)

23. Gubbay, J. et al. Inverted repeat structure of the Sry locus in mice. Proc Natl Acad Sci U S A 89, 7953-7957 (1992).

24. Obata, Y. et al. Post-implantation development of mouse androgenetic embryos produced by in-vitro fertilization of enucleated oocytes. Hum Reprod 15, 874-80 (2000).

25. Orikasa, C. \& Sakuma, Y. Estrogen configures sexual dimorphism in the preoptic area of C57BL/6J and ddN strains of mice. J Comp Neurol 518, 3618-3629 (2010).

26. Bowles, J., Cooper, L., Berkman, J. \& Koopman, P. Sry requires a CAG repeat domain for male sex determination in Mus musculus. Nat Genet 22, 405-408 (1999).

27. Battiloro, E. et al. A novel double nucleotide substitution in the HMG box of the SRY gene associated with Swyer syndrome. Hum Genet 100, 585-587 (1997)

28. Veitia, R. et al. Mutations and sequence variants in the testis-determining region of the Y chromosome in individuals with a 46,XY female phenotype. Hum Genet 99, 648-652 (1997).

29. Harley, V. R. et al. Defective importin beta recognition and nuclear import of the sex-determining factor SRY are associated with XY sex-reversing mutations. Proc Natl Acad Sci U S A 100, 7045-7050 (2003).

30. Sim, H. et al. Defective calmodulin-mediated nuclear transport of the sexdetermining region of the $\mathrm{Y}$ chromosome (SRY) in XY sex reversal. Mol Endocrinol 19, 1884-1892 (2005).

31. Harley, V. R. \& Goodfellow, P. N. The biochemical role of SRY in sex determination. Mol. Reprod Dev 39, 184-193 (1994).

32. Killick, S. \& Elstein, M. Phamacologic production of lutenized unruptured follicles by prostaglandin synthetase inhibitors. Fertil Steril 47, 773-777 (1987).

33. Marik, J. \& Hulka, J. Lutenized unruptured follicle syndrome; a subtle cause of infertility. Fertil Steril 29, 270-274 (1978).

34. Hamilton, C. J. et al. Follicle growth curves and hormonal patterns in patients with the lutenized unruptured follicle syndrome. Fertil Steril 43, 541-548 (1985).

35. LeMaire, G. S. The lutenized unruptured follicle syndrome: anovulation in disguise. J. Obstet. Gynecol. Neonatal Nurs 16, 116-120 (1987).

36. Mathieson, W. B., Taylor, S. W., Marshall, M. \& Neumann, P. E. Strain and sex differences in the morphology of the medial preoptic nucleus of mice. J Comp Neurol 428, 254-265 (2000).
37. Messager, S. et al. Kisspeptin directly stimulates gonadotropin-releasing hormone release via G protein-coupled receptor 54. Proc. Natl Acad Sci U S A 102, 1761-1766 (2005)

38. Keller, M., Pierman, S., Douhard, Q., Baum, M. J. \& Bakker, J. The vomeronasal organ is required for the expression of lordosis behaviour, but not sex discrimination in female mice. Eur J Neurosci 23, 521-530 (2006).

39. Anderson, G. M. et al. Evidence that thyroid hormones act in the ventromedial preoptic area and the premammillary region of the brain to allow the termination of the breeding season in the ewe. Endocrinology 144, 2892-2901 (2003).

40. Anderson, G. M., Connors, J. M., Hardy, S. L., Valent, M. \& Goodman, R. L. Oestradiol microimplants in the ventromedial preoptic area inhibit secretion of luteinizing hormone via dopamine neurones in anoestrous ewes. J Neuroendocrinol 13, 1051-1058 (2001).

41. Rosser, A. E., Remfry, C. J. \& Keverne, E. B. Restricted exposure of mice to primer pheromones coincident with prolactin surges blocks pregnancy by changing hypothalamic dopamine release. J Reprod Fertil 87, 553-559 (1989).

42. Gregg, C., Zhang, J., Butler, J. E., Haig, D. \& Dulac, C. Sex-specific parent-of-origin allelic expression in the mouse brain. Science 329, 682-685 (2010).

43. Sakuma, T. et al. Efficient TALEN construction and evaluation methods for human cell and animal applications. Genes Cells 18, 315-326 (2013).

44. Cermak, T. et al. Efficient design and assembly of custom TALEN and other TAL effector-based constructs for DNA targeting. Nucleic Acids Res 39, e82 (2011).

\section{Acknowledgments}

We would like to thank Maki Fukami for thoughtful discussion and critical reading of the manuscript, Yuko Katoh-Fukui and Kenji Miyado for helpful discussion and Mami Miyado for instructions regarding AGD measurement. This work was supported, in part, by The Grant of National Center for Child Health and Development, Grant Number 25-1, JSPS KAKENHI Grant Number 24115707 and JST (CREST) for H.A. and JSPS KAKENHI Grant Number 25132713 and MEXT KAKENHI Grant Number 23570265 and The Grant of National Center for Child Health and Development, Grant Number 24-3 for S.T.

\section{Author contributions}

K.Miyata, S.Y., S.M. and S.T. constructed the TALEN plasmids, T.S. and T.Y. provided instructions regarding the construction of the TALEN plasmids, M.T. performed the microinjection experiments, M.S. and T. Kikusi carried out the detection of the dimorphisms in the brain, S.Y., M.I., K.Miura. and Y.K. performed detection of SRY protein, T. Kato and S.T. conducted the remainder of the experiments, M.I., H.A. and S.T. designed the project and S.T. wrote the paper. All authors discussed the results and commented on the manuscript.

\section{Additional information}

Competing financial interests: The authors declare no competing financial interests.

How to cite this article: Kato, T. et al. Production of Sry knockout mouse using TALEN via oocyte injection. Sci. Rep. 3, 3136; DOI:10.1038/srep03136 (2013).

cc) (i) $\Theta$ This work is licensed under a Creative Commons Attribution-

NonCommercial-NoDerivs 3.0 Unported license. To view a copy of this license, visit http://creativecommons.org/licenses/by-nc-nd/3.0 